\title{
Dynamical Diffraction S/TEM Simulations from Molecular Dynamics Data
}

\author{
Joseph Tessmer ${ }^{1}$, Saransh Singh ${ }^{1}$ and Marc De Graef ${ }^{1}$
}

${ }^{1}$ Dept. of Materials Science and Engineering, Carnegie Mellon Univ., Pittsburgh PA 15213, USA

Transmission Electron Microscopy is a powerful technique for the characterization of defects in crystalline materials, operating in either the conventional parallel-beam modality or the scanning convergedbeam modality. Understanding defect behavior in materials is critical because of the large impact they can have on performance. Typically, identifying contrast features of defects from experimental images can be difficult and often requires detailed image simulations, in particular in the case of STEM illumination. Historically, these simulations have relied on the availability of analytical expressions for the defect displacement field in a perfect crystal. However, many complex defects, oftentimes in multi-phase material systems, cannot be represented by closed-form analytical expressions, limiting the traditional image simulation approach. Therefore, there is a growing need for the integration of material simulation techniques with defect image simulations to address these challenging materials characterization problems.

In this contribution, we introduce a dynamical diffraction simulation method which takes as input a set of atom coordinates that define the crystal to be imaged. This atom coordinate data is often itself the result of a molecular dynamics (MD) simulation, a method which calculates the trajectories of atoms and molecules by integrating Newton's equations of motion for interacting bodies. The S/TEM simulation makes use of a physics-based forward model described in detail in [1]. The forward model uses the scattering matrix formalism together with the column approximation to simulate electronsample interaction by separating the volume occupied by the atoms into columns of cells. The relevant scattering matrix equation is given by:

$$
\frac{\mathrm{d} \mathbf{S}(z)}{\mathrm{d} z}=\mathrm{i} \mathcal{A} \mathbf{S}(z) \quad \rightarrow \quad \mathbf{S}\left(z_{0}\right)=\mathrm{e}^{\mathrm{i} \mathcal{A} z_{0}} \mathbf{S}(0)=\mathcal{S}(z) \mathbf{S}(0)
$$

where $\mathcal{A}$ is the structure matrix, $\mathcal{S}(z)$ is the scattering matrix, which relates the initial electron wave amplitude $\mathbf{S}(0)$ to $\mathbf{S}(z)$, the electron wave amplitude at depth $z$. For each cell in a column, the scattering matrix is computed for a select set of reciprocal lattice reflections, $\mathbf{g}$, followed by the propagation of the beam down the column. A final image in conventional TEM mode is compiled by taking the final beam intensity from the bottom of each column and using these intensity values to form an image.

This atom-coordinate based simulation method differs from the conventional dynamical diffraction simulation method, which assumes that the crystal lattice is perfect. In the conventional method, a defect image can be produced using the perfect lattice along with an analytic expression for the defect. The atomistic method introduced here differs by accounting for the dislocation directly in the atom coordinates. Additionally, the atomistic simulation uses cells in which the atoms do not lie perfectly on the lattice sites. As a result, any defect that can be represented by changes in the atom coordinates can be simulated using the atomistic approach. These defects can be introduced using simulation methods such as molecular dynamics, and present a more realistic view of defect behavior.

For the STEM modality, several modifications must be made to the simulation due to computation time concerns. While the main computation loop is parallelized per-column, completely recomputing the scattering matrix for each slice is time-consuming. When we consider a converged probe, where a scattering matrix must be computed not only for each slice but for each beam direction, this becomes 
prohibitively expensive. Rather than recompute the entire structure matrix for all beam directions for a given cell, we can split the matrix into two components: the off-diagonal entries, which describe the coupling between beams, and the diagonal entries, which describe the excitation errors. For a small beam tilt such as those used in STEM, only the diagonal part changes, and we split the structure matrix into two parts $\mathcal{A}_{n}=A_{n}+B_{\kappa}$, where $A_{n}$ is the off-diagonal part and $B_{\kappa}$ a diagonal matrix containing the excitation errors and normal absorption for the incident beam direction labeled by $\kappa$. This approach results in significant time savings compared to re-computation of the complete structure matrix.

Fig. 1(a) shows a comparison of a defect image computed using the atom coordinate approach on the left and one computed from atom coordinates on the right with excellent agreement between the two approaches. Experimental evidence suggests that the width of stacking faults in High Entropy Alloys (HEA) such as CrMnFeCoNi show spatial variation. This has been attributed to the hyper-local compositional variation in such alloy systems. Molecular dynamics simulations were performed for a stacking fault in a representative FeNiCr alloy. Fig. 1(b) shows an example of several simulated BF/DF TEM images calculated using the proposed approach; the DF images are labeled to indicate the corresponding $\mathbf{g}$ vector. The [113] reflection is shown enlarged with intensity profiles for several sections of the dislocation showing spatially varying stacking fault widths.

\section{References:}

[1] P.J. Philips, M.J. Mills, and M. De Graef, Philosophical Magazine, 91(16):2081-2101.

[2] The authors acknowledge M. Ghazisaeidi (OSU) for providing the MD data set, an ONR Vannevar Bush Faculty Fellowship (N00014-16-1-2821), and the computational resources of the Materials Characterization Facility at CMU, grant MCF-677785.

\section{Figure 1}

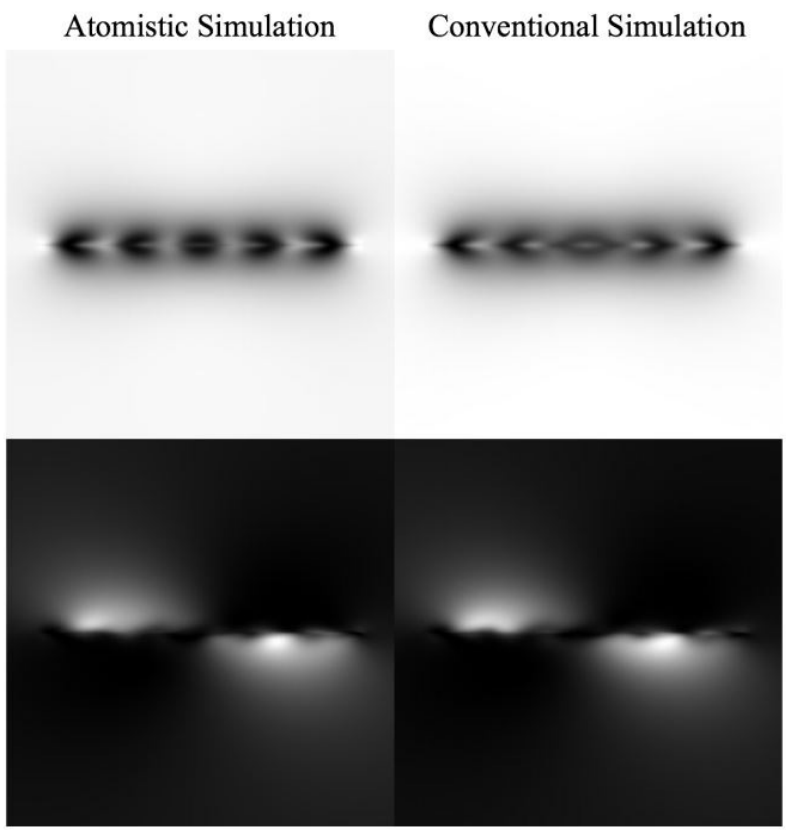

(a) Comparison of simulation approaches
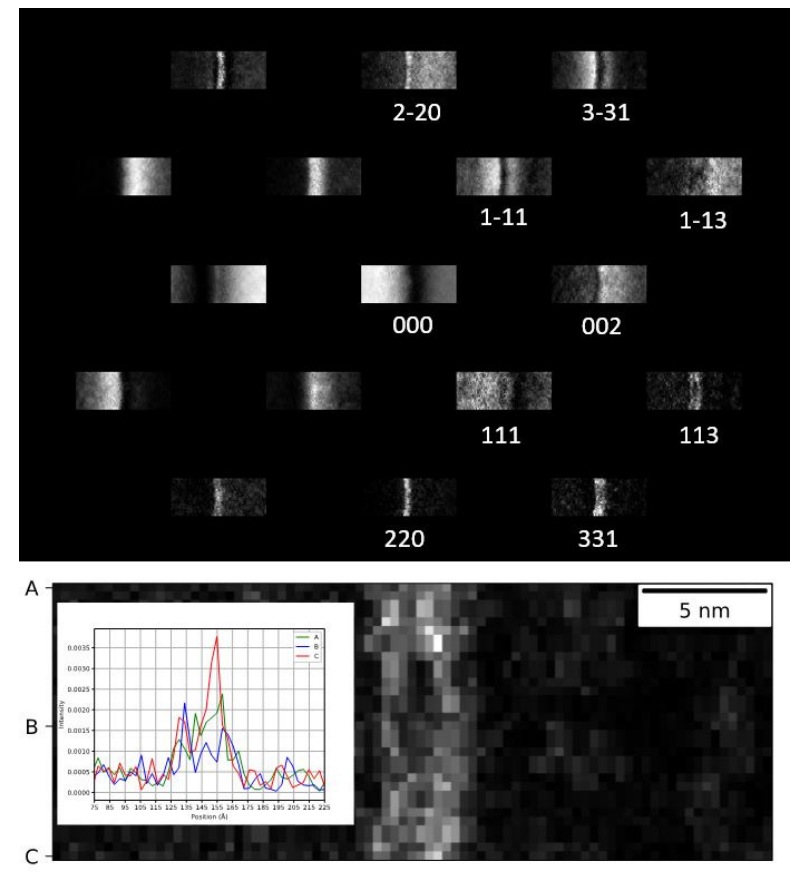

(b) Top: Several BF/DF simulated images for a HEA Bottom: [113] DF image with intensity profiles 